\title{
Genetic diversity of Plasmodium falciparum among school-aged children from the Man region, western Côte d'Ivoire
}

\author{
Sarah E Mara ${ }^{1,2}$, Kigbafori D Silué ${ }^{1,2}$, Giovanna Raso ${ }^{2,3,4}$, Simon P N'Guetta', Eliézer K N'Goran 1,2, Marcel Tanner ${ }^{3,4}$, \\ Jürg Utzinger ${ }^{3,4}$ and Xavier C Ding ${ }^{2^{*}}$
}

\begin{abstract}
Background: The genetic diversity of Plasmodium falciparum allows the molecular discrimination of otherwise microscopically identical parasites and the identification of individual clones in multiple infections. The study reported here investigated the $P$. falciparum multiplicity of infection (MOI) and genetic diversity among school-aged children in the Man region, western Côte d'Ivoire.

Methods: Blood samples from 292 children aged seven to 15 years were collected in four nearby villages located at altitudes ranging from 340 to $883 \mathrm{~m}$ above sea level. Giemsa-stained thick and thin blood films were prepared and examined under a microscope for $P$. falciparum prevalence and parasitaemia. $\mathrm{MOI}$ and genetic diversity of the parasite populations were investigated using $m s p 2$ typing by polymerase chain reaction-restriction fragment length polymorphism (PCR-RFLP).

Results: Plasmodium falciparum prevalence and parasitaemia were both found to be significantly lower in the highest altitude village. Genotyping of the isolates revealed 25 potentially new msp2 alleles. MOI varied significantly across villages but did not correlate with altitude nor children's age, and only to a limited extent with parasitaemia. An analysis of molecular variance (AMOVA) indicated that a small, but close to statistical significance $(p=0.07)$, fraction of variance occurs specifically between villages of low and high altitudes.

Conclusions: Higher altitude was associated with lower prevalence of $P$. falciparum but not with reduced $\mathrm{MOI}$, suggesting that, in this setting, $\mathrm{MOI}$ is not a good proxy for transmission. The evidence for partially parted parasite populations suggests the existence of local geographical barriers that should be taken into account when deploying anti-malarial interventions.
\end{abstract}

Keywords: Plasmodium falciparum, msp2, Genetic diversity, PCR-RFLP, Côte d'Ivoire

\section{Background}

The widespread genetic diversity of Plasmodium falciparum populations plays an important role on several aspects of parasite biology, and consequently on the efficacy of anti-plasmodial interventions. For example, polymorphic elements and genetic plasticity contribute to modulate malaria morbidity and mortality and play a key role in P. falciparum immune evasion and the parasite's adaptation to evolving environmental conditions such as drug pressure $[1,2]$. This highly polymorphic nature can

\footnotetext{
* Correspondence: xavier.ding@gmail.com

2Département Environnement et Santé, Centre Suisse de Recherches Scientifiques en Côte d'Ivoire, 01 BP 1303 Abidjan 01, Côte d'Ivoire Full list of author information is available at the end of the article
}

also be harnessed to study the structure and dynamics of parasite populations (see, for example, references [3,4]) and molecular investigations in epidemiological studies and clinical trials, allowing discrimination between re-infection and recrudesce [5]. This is typically achieved by typing one or more of three highly polymorphic genes: msp1 (merozoite surface protein 1), msp2 (merozoite surface protein 2), and glurp (glutamate-rich protein), which encode antigenic proteins of poorly characterized functions [6].

Studies pertaining to the genetic diversity of $P$. falciparum have been essentially comparing either very distant locations (e.g. intercontinental or spread within one or several countries, see [7-11]), or a small number of 
epidemiologically very distinct locations [12-15] and reported observations on the complex interplay between transmission intensity, the multiplicity of infection (MOI) with Plasmodium and the genetic structure of local parasite populations. High transmission settings have been frequently, but not always, associated with greater genetic diversity and MOI $[16,17]$.

A large study of the demographic, environmental and socioeconomic risk factors associated with malaria, schistosomiasis and soil-transmitted helminthiasis was previously conducted in 57 villages of the mountainous region of Man in western Côte d'Ivoire [18-21]. The local topography is an extension of the Fouta Djallon highland area and is characterized by rounded mountains in the north, ranging in altitudes from 200 to $1,300 \mathrm{~m}$ above sea level, and an abrupt transition to plains in the south. The area is hyperendemic for P. falciparum infection and polyparasitism (concurrent infection with different species of helminths, intestinal protozoa and Plasmodium) is very common [22]. In the work presented here, the MOI with $P$. falciparum is further explored. Additionally, the genetic diversity of the local $P$. falciparum populations is assessed for a subset of the previously collected samples. Specifically, four villages located in close proximity to each other but at various altitudes have been selected (Figure 1). Emphasis is placed on a small-scale comparison of $P$. falciparum msp 2 allelic diversity in close but geographically contrasted areas of western Côte d'Ivoire. The importance of the findings in the context of the current malaria elimination and eradication agenda [23] is discussed.

\section{Methods}

\section{Ethical consideration}

The study protocol was approved by the institutional research commission of the Swiss Tropical and Public Health Institute (Basel, Switzerland) and the Centre Suisse de Recherches Scientifiques en Côte d'Ivoire (Abidjan, Côte d'Ivoire). Ethical clearance was obtained from the Ministry of Health in Côte d'Ivoire. Regional health and education authorities, village leaders (chief, school director and teachers) and parents of school-going children were informed about the purpose and procedures of the study. Written informed consent was obtained from parents/ legal guardians, whereas children assented orally. Participation was voluntary and children could withdraw anytime without further obligations.

\section{Study sites and population}

The study sites are located in the Man region in western Cote d'Ivoire, where P. falciparum is hyperendemic [21]. It is a humid forest area with a dense river network that drains the entire region [25]. The northern part of the study area is mountainous whereas the southern part is a plain.

Between October 2001 and February 2002, 57 villages of this area were investigated to study the spatial distribution of infections with Plasmodium spp. [20,21], Schistosoma mansoni $[18,25]$, and hookworm [19] among school-aged children. For the present study, a subset of four villages was selected, namely (i) Bogouiné II (geographical coordinates: $709.000 \mathrm{~N}$ latitude, $745.000 \mathrm{~W}$ longitude, $340 \mathrm{~m}$ above sea level); (ii) Bloleu (6 $52.250 \mathrm{~N}, 816.333 \mathrm{~W}$,
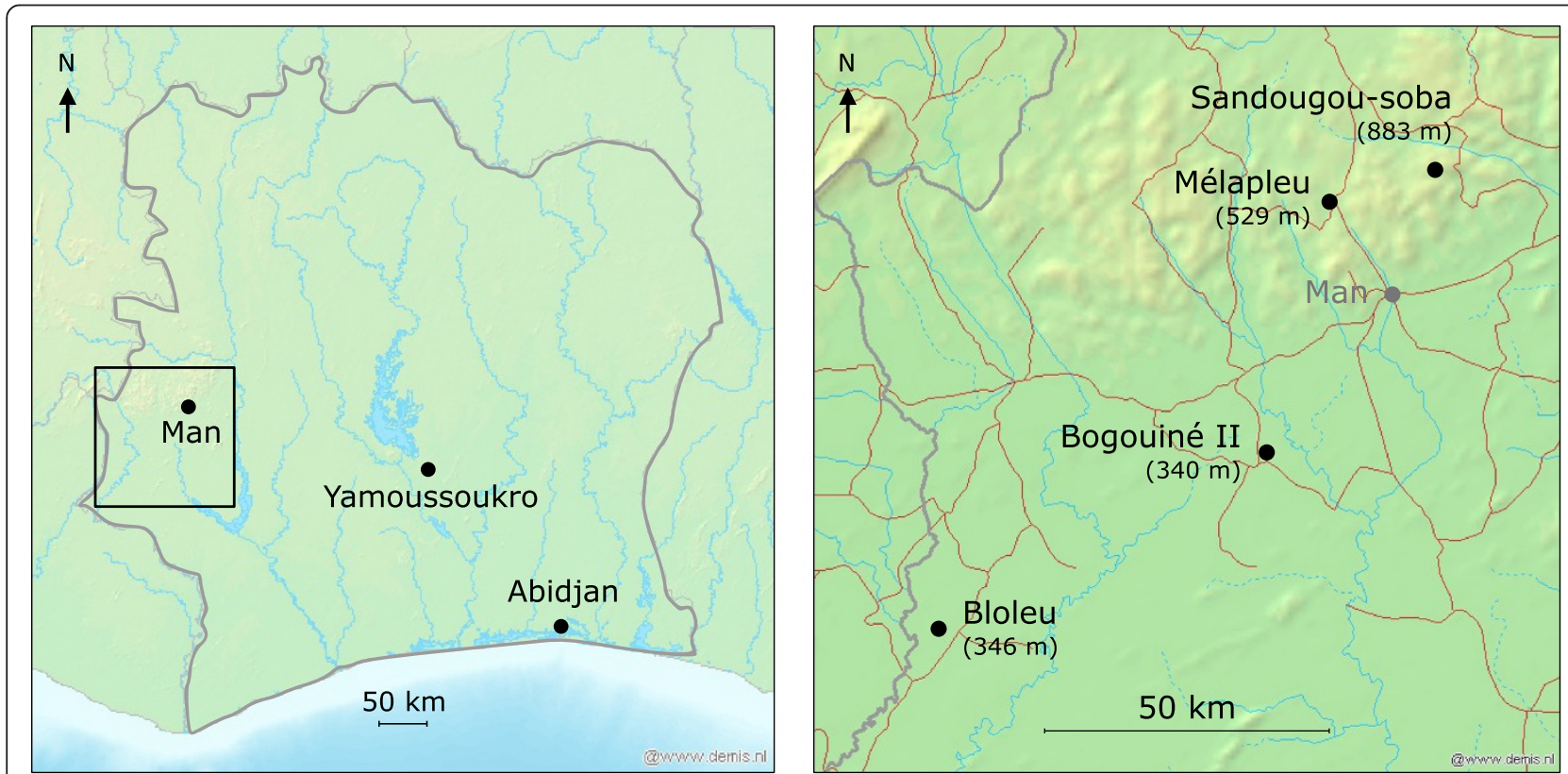

Figure 1 Study sites location. Four villages located in the Man region, western Côte d'Ivoire, were selected for this study. Bogouiné II and Bloleu are in a plain at relatively low altitude (340 and $346 \mathrm{~m}$ above sea level, respectively). Mélapleu and Sandougou-soba are in a mountainous area at relatively high altitude (529 and 883 m, respectively). Maps have been generated using GPS Visualizer [24]. 
346 m); (iii) Mélapleu (7 32.800 N, 739.000 W, 529 m); and (iv) Sandougou-soba (7 35.833 N, 728.916 E, 883 m). The villages are located in a close range, at $120 \mathrm{~km}$ maximal distance, but in contrasting geographical settings with a maximal altitude difference of approximately $540 \mathrm{~m}$. Mélapleu and Sandougou-soba are located northwest of Man, in the mountainous area, whereas Bogouiné II and Bloleu are located south of Man in a plain (Figure 1).

As described in detail elsewhere [18], all primary school children attending grades 3-5 were invited to participate. The study population in the four selected villages consisted of 292 school-going children, aged between seven and 15 years. Anti-malarial treatments were given to children with malaria-related symptoms (e.g. headache) and axillary temperature $\geq 37.5^{\circ} \mathrm{C}$. Children found with helminth eggs in their stool were given anthelminthic drugs (i.e. praziquantel, $40 \mathrm{mg} / \mathrm{kg}$ against $S$. mansoni; albendazole, $400 \mathrm{mg}$ against soil-transmitted helminths) $[18,19]$.

\section{Sampling and molecular analysis}

Finger-prick whole blood samples were obtained from each child. Thick and thin blood films were prepared on microscope slides and air-dried. Additionally, approximately $200 \mu \mathrm{l}$ of blood was collected into microtainer tubes. Samples were transferred to a laboratory in the town of Man. Slides were stained with $10 \%$ Giemsa and forwarded to a laboratory in Abidjan, where they were examined under a microscope by experienced laboratory technicians for Plasmodium species identification and parasitaemia, assuming a count of 8,000 leukocytes per $\mu$ l of blood [20,21]. Microtainers were stored at $-20^{\circ} \mathrm{C}$ pending molecular analyses.

Blood samples were thawed on ice and used for Plasmodium DNA extraction, $m s p 2$ nested polymerase chain reaction (PCR) amplification and analysis by restriction fragment length polymorphism (RFLP), as described elsewhere [26-28]. Briefly, $10 \mu \mathrm{l}$ of whole blood were washed twice in cold sodium phosphate buffer (5 mM, pH 8.0), boiled for $10 \mathrm{~min}$ in $50 \mu \mathrm{l}$ of sterile $\mathrm{ddH}_{2} \mathrm{O}$ and centrifuged at $14,000 \mathrm{~g}$ for $10 \mathrm{~min}$. Next, $5 \mu \mathrm{l}$ of the supernatant were used for the $P$. falciparum $m s p 2$ nested PCR reactions. The presence of PCR amplification products was determined using ethidium bromide stained 1\% agarose gel photographed under UV illumination. PCR amplification products were digested with HinfI restriction enzymes. Restriction digest products were resolved on a $10 \%$ acrylamide gel subsequently stained with ethidium bromide and photographed under UV illumination.

The size of restriction fragments was determined using the ImageJ software [29] together with the MolWT macro (supplied by PHASE GmbH; Luebeck, Germany). Allelic determination was performed as recommended previously and using $10 \mathrm{bp}$ bins for the 3D7 variable fragment [28].

\section{Statistical analysis}

The data were entered into Microsoft Excel 2007 and analysed using Prism version 6.0c or GenAlEx version 6.5 $[30,31]$. The number of effective alleles was calculated as $\left[1 / \Sigma p i^{2}\right]$, the Shannon's information index as $\left[-1^{*} \Sigma\left(p i^{*} \mathrm{Ln}\right.\right.$ $(p i)]$, the haploid genetic diversity as $\left[1-\Sigma p i^{2}\right]$, and the theoretical probability of two clones to share the same genotype as $\left[\Sigma p i^{2}\right]$, where $\mathrm{n}$ is the sample size and $p i$ the frequency of allele $i$.

\section{Results}

\section{Plasmodium falciparum prevalence and parasitaemia}

Overall, 292 blood samples from four selected villages were analysed by PCR for the presence of $P$. falciparum parasites. A total of 241 samples were found positive, resulting in a prevalence of $82.5 \%$, which is slightly higher than the prevalence originally determined by microscopy, at $78.8 \%$ (see Additional file 1). For all subsequent analyses, the 27 samples found positive by PCR but negative by microscopy, were assigned a parasitaemia of four parasites/ $\mu \mathrm{l}$ of blood. The prevalence in Bogouiné II, Bloleu and Mélapleu were $87.0 \%, 89.7 \%$ and $88.5 \%$. A significantly lower prevalence was found in Sandougou-soba (57.4\%), the village at the highest altitude $\left(p<0.01, \mathrm{X}^{2}\right.$-test).

Parasitaemia, as determined by microscopy and corrected as mentioned above, was also found to be significantly different across villages ( $p=0.039$, Kruskal-Wallis test), with geometric means (GMs) ranging from 80.1 to 331.6 parasite/ $\mu \mathrm{l}$ of blood (Table 1 ). Similarly to prevalence, the lowest parasitaemia GM was noted in the village located at the highest altitude, Sandougou-soba.

\section{msp2 genotyping and multiplicity of infection}

In order to better define the epidemiological profiles of the four study villages, a detailed $m s p 2$ genotyping analysis was carried out, using RFLP on the 241 PCR-positive samples. Of note, $m s p 2$ alleles are generally grouped in two distinct families, the FC27 type and the 3D7 type, which are characterized by the presence of specific repetitive elements. Moreover, a large diversity of alleles exists within each family type and these can be readily distinguished by analysing the size of fragments obtained after a HinfI digestion (Figure 2A, B). FC27-type alleles are characterized by two fragments of fixed size, $115 \mathrm{bp}$ and $137 \mathrm{bp}$, together with a combination of fragments of variable size, depending on the specific pattern of repeats. 3D7-type alleles are characterized by two fixed size fragments of $70 \mathrm{bp}$ and $108 \mathrm{bp}$ and a third fragment of variable size [28]. A public domain image processing software was employed to determine the size of the HinfI fragments obtained from each sample after separation on polyacrylamide gels (Figure 2C, D) [29]. To evaluate the precision of the size determination method, the measures obtained with fixed-size fragments, that is the $115 \mathrm{bp}$ and $137 \mathrm{bp}$ 
Table 1 Plasmodium falciparum prevalence and parasitaemia at the study sites

\begin{tabular}{|c|c|c|c|c|c|c|}
\hline Village & $\begin{array}{l}\text { Altitude } \\
\text { ( } \mathrm{m} \text { above sea level) }\end{array}$ & $\begin{array}{l}\text { Sampling date } \\
\text { (dd.mm.yyyy) }\end{array}$ & $\mathrm{n}$ & $\mathrm{Age}^{\mathrm{a}}$ & P. falciparum-infected $(\%)^{b}$ & Parasitaemia $(95 \% \mathrm{Cl})^{\mathrm{c}}$ \\
\hline Sandougou-soba & 883 & 13.12 .2001 & 54 & $10.2(1.5)$ & $31(57.4)$ & $80.1(31.3-204.7)$ \\
\hline Mélapleu & 529 & 29.11 .2001 & 78 & $10.1(1.9)$ & $69(88.5)$ & $207.6(135.1-319.3)$ \\
\hline Bloleu & 346 & 07.02 .2002 & 68 & $10.0(1.1)$ & $61(89.7)$ & $227.3(138.9-371.8)$ \\
\hline Bogouiné II & 340 & 29.01 .2002 & 92 & $10.1(1.1)$ & $80(87.0)$ & $331.6(220.8-498.0)$ \\
\hline
\end{tabular}

Average in years (SD).

${ }^{b}$ Number of $P$. falciparum-infected children, $p<0.01\left(x^{2}\right)$.

'Geometric mean of parasites/ $\mu$ l of blood, $p=0.039$ (Kruskal-Wallis test).

fragments from FC27-type alleles and the $70 \mathrm{bp}$ and 108 bp ones from 3D7-type alleles, were used. Altogether, the averages of the measured fragments did not deviate of more than $3 \mathrm{pb}$ from the expected sizes, with standard deviations all under $4 \mathrm{bp}$ (see Additional file 2). It was thus considered reasonable to pool fragments of variable size in bins of $10 \mathrm{bp}$. When possible, the nomenclature reported previously for specific FC27 alleles was used, whilst the 3D7 alleles are named according to the bin size of the variable fragment.

A total of 85 distinct $m s p 2$ alleles, 45 of the FC27-type and 40 of the 3D7-type, were identified from the 241 blood samples analysed (Table 2). Two recombinant alleles were also identified (FC27 REC1 and FC27 REC2) and classified as FC27-type. Eighteen of the 45 FC27-type alleles identified appear to have not yet been reported elsewhere and some of these display insertion or deletion in the $137 \mathrm{bp}$ fragment. Similarly, seven of the 40 3D7-types alleles display an unusually short variable fragment $(<300 \mathrm{bp})$ or an additional $45 \mathrm{bp}$ fragment. These 25 potentially newly identified $m s p 2$ alleles have been named Cot 7 to Cot 31 , following the nomenclature used for $m s p 2$ allele previously identified in Côte d'Ivoire [32]. The details of the specific Hinfl fragments are indicated in Table 3.

Overall, 694 distinct $P$. falciparum infections were recorded from the 241 PCR-positive samples analysed. MOI, that is the number of clonal infection per individual, ranges from 1 to 8 , with a mean of 2.88 (Table 2). The mean MOI were found to be significantly different across the study sites with values ranging from 2.32 to 3.42 (Table 2). This difference occurs between the geographically related villages and does not seem to correlate with

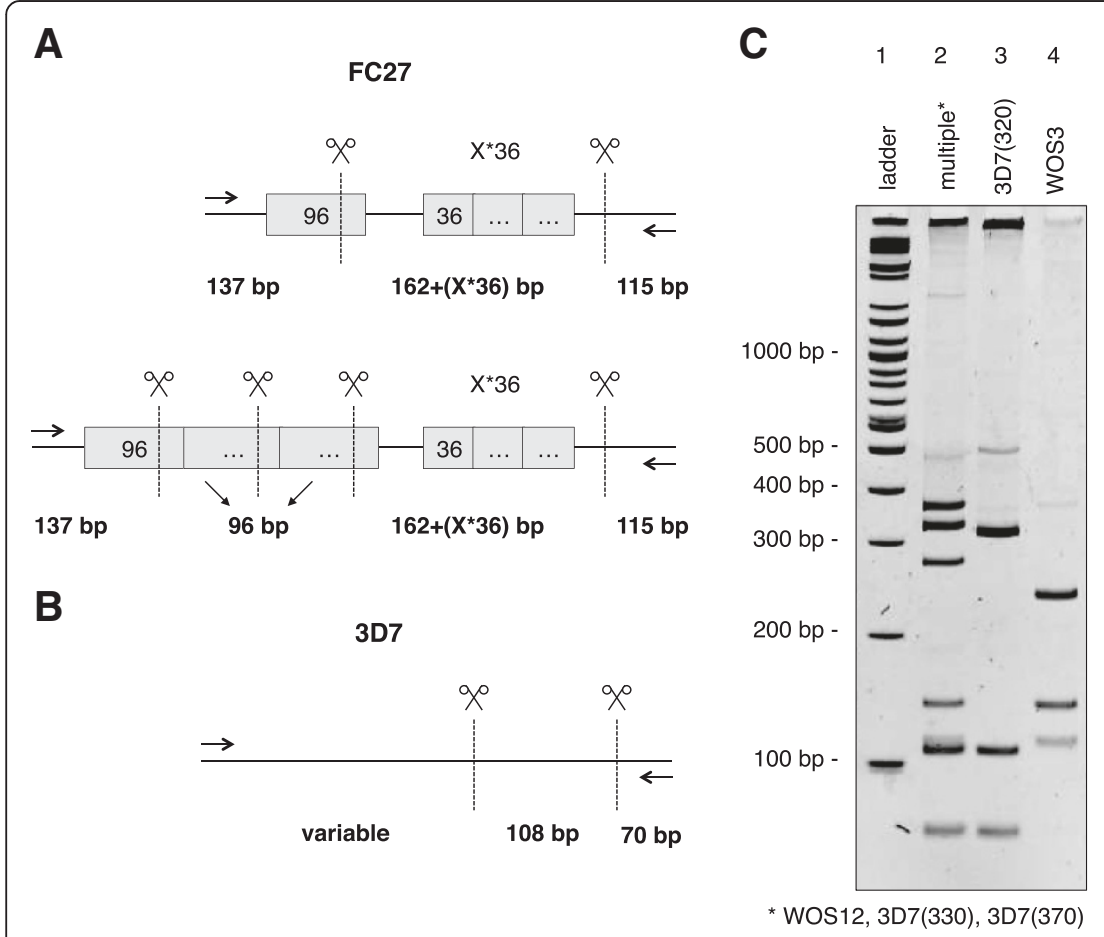

D

Figure 2 msp2 genotyping by PCR-RFLP. Hinfl restriction digest pattern of (A) FC-27 type alleles and (B) 3D7-type alleles. The sizes of fixed fragments are indicated, repetitive elements are indicated as grey boxes with sizes in bp. (C) Typical polyacrylamide gel electrophoresis result. Lane 2 contains a mixture of one FC27-type allele (WOS12) and two 3D7-type allele (330 and 370). Lanes 3 and 4 contain a single msp2 allele. (D) ImageJ fragment size analysis of the gel displayed in panel C. The 3D7 108 bp fragment and the FC27 115 bp fragment co-migrate as a single peak in case of mixed sample (lane 2). 
Table 2 msp2 allele type

\begin{tabular}{lllllll}
\hline Village & $\mathbf{n}$ & $\mathbf{N a}^{\mathbf{a}}$ & FC27-type & 3D7-type & P. falciparum infection & Mean MOI (SD) $^{\mathbf{b}}$ \\
\hline Sandougou-soba & 31 & 39 & 14 & 25 & 72 & $2.32(1.33)$ \\
Mélapleu & 69 & 58 & 30 & 28 & 235 & $3.41(1.75)$ \\
Bloleu & 61 & 58 & 27 & 31 & 201 & $3.29(1.72)$ \\
Bogouiné II & 80 & 60 & 26 & 34 & 186 & $2.32(1.38)$ \\
Total & 241 & 85 & 45 & 40 & 694 & $2.88(1.65)$ \\
\hline
\end{tabular}

${ }^{\mathrm{a}}$ Number of distinct alleles.

${ }^{\mathrm{b}} p<0.001$ (Kruskal-Wallis test on site-specific MOls).

the site-specific $P$. falciparum prevalence or mean parasitaemia. For instance, the highest parasitaemia GM (425.0) was observed in Bogouiné II, where the lowest mean MOI was found (2.32).

No link between MOI and participants' age could be identified when considering the whole study population or each of the four villages separately (Table 4 and Additional file 3). A limited, but statistically significant, link between MOI and parasitaemia was observed (Table 4). MOI is significantly lower in the first parasitaemia quartile (four to

Table 3 Details of potentially new msp2 alleles

\begin{tabular}{|c|c|c|c|c|c|}
\hline \multirow{2}{*}{$\begin{array}{l}\text { Name } \\
\text { Cot7 }\end{array}$} & \multirow{2}{*}{$\begin{array}{l}\text { Family type } \\
\text { FC27 }\end{array}$} & \multicolumn{4}{|c|}{ Hinf I fragment sizes ${ }^{a}$} \\
\hline & & 115 & 137 & $\sim 244$ & \\
\hline Cot8 & FC27 & 115 & 137 & $\sim 320$ & \\
\hline Cot10 & FC27 & 115 & 137 & $\sim 228$ & \\
\hline Cot11 & FC27 & 115 & 137 & $\sim 280$ & \\
\hline Cot13 & FC27 & 115 & $\sim 131$ & $\sim 320$ & \\
\hline Cot15 & FC27 & 115 & 137 & $\sim 191$ & \\
\hline Cot14 & FC27 & 115 & $\sim 120$ & $\sim 227$ & \\
\hline Cot17 & FC27 & 115 & 137 & $\sim 440$ & \\
\hline Cot19 & FC27 & 115 & 137 & $\sim 396$ & \\
\hline $\operatorname{Cot} 20$ & FC27 & 115 & 137 & $\sim 404$ & \\
\hline $\operatorname{Cot} 21$ & FC27 & 115 & 137 & $\sim 454$ & \\
\hline $\operatorname{Cot} 22$ & FC27 & 115 & 137 & $\sim 207$ & \\
\hline Cot24 & FC27 & 115 & 137 & $\sim 430$ & \\
\hline Cot25 & FC27 & 115 & 137 & $\sim 415$ & \\
\hline Cot26 & FC27 & 115 & 137 & $\sim 357$ & \\
\hline Cot29 & FC27 & 115 & 137 & $\sim 333$ & \\
\hline $\operatorname{Cot} 30$ & FC27 & 115 & 137 & $\sim 180$ & \\
\hline Cot31 & FC27 & 115 & $\sim 198$ & $\sim 237$ & \\
\hline Cot9 & 3D7 & & 70 & 108 & $\sim 153$ \\
\hline Cot12 & 3D7 & & 70 & 108 & $\sim 236$ \\
\hline Cot16 & 3D7 & & 70 & 108 & $\sim 190$ \\
\hline Cot18 & 3D7 & $\sim 45$ & 70 & 108 & $\sim 490$ \\
\hline Cot23 & 3D7 & & 70 & 108 & $\sim 180$ \\
\hline Cot27 & 3D7 & $\sim 45$ & 70 & 108 & $\sim 300$ \\
\hline Cot28 & 3D7 & $\sim 45$ & 70 & 108 & $\sim 460$ \\
\hline
\end{tabular}

${ }^{\mathrm{a}}$ Exact sizes of fixed fragments are given, $\sim$ indicates measured sizes.
72 parasites/ $\mu$ l of blood) with a mean of 2.02, while it is comparable across the second, third and fourth quartiles with mean MOI values ranging between 3.12 and 3.23.

\section{Plasmodium falciparum genetic diversity}

Plasmodium falciparum prevalence and parasitaemia, contrary to MOI, appear to be linked to some extent with the geographical location of the study sites. To further explore the specificity of the investigated sites, the sitespecific $P$. falciparum genetic diversities were assessed.

Figure 3 displays the allelic frequencies observed at the respective study sites as well as when pooling all samples. The most prevalent allele in each village is the FC27-type allele WOS3, with a maximum local frequency of $11.1 \%$. The second most prevalent allele in the two highest villages, Sandougou-soba and Mélapleu, is WOS10 (6.9\% and $7.2 \%$, respectively), while it is 3D7 (400) in Bogouiné II and the newly reported FC27-type allele Cot11 in Bloleu. These two alleles rank amongst the 18th and 9th most prevalent in the other locations, respectively. Between one and nine private alleles were identified in each village, but their local frequency did not exceed $1.4 \%$ (Figure 3 ). The lowest number of distinct msp2 alleles (39) was observed in Sandougou-soba, which is likely due to the lower number of individual infections sampled in this village as compared to the others (72 versus 186 to 235) [33] (Table 5). Nevertheless, 60 different alleles were identified out of 186 clonal infections in the low altitude

Table 4 Relationship between MOI and age or parasitaemia

\begin{tabular}{|c|c|c|c|c|}
\hline Variable & Stratification & $n$ & MOI (SD) & $p$ value \\
\hline \multirow[t]{2}{*}{ Age group } & 7-10 years & 150 & $2.95(1.76)$ & $0.77^{a}$ \\
\hline & 11-15 years & 91 & $2.78(1.47)$ & \\
\hline \multirow[t]{4}{*}{ Parasitaemia } & 1st quartile & 60 & $2.02(1.13)$ & $<0.001^{c}$ \\
\hline & 2nd quartile & 60 & $3.17(1.80)$ & \\
\hline & 3rd quartile & 61 & $3.23(1.65)$ & \\
\hline & 4th quartile & 60 & $3.12(1.70)$ & \\
\hline
\end{tabular}




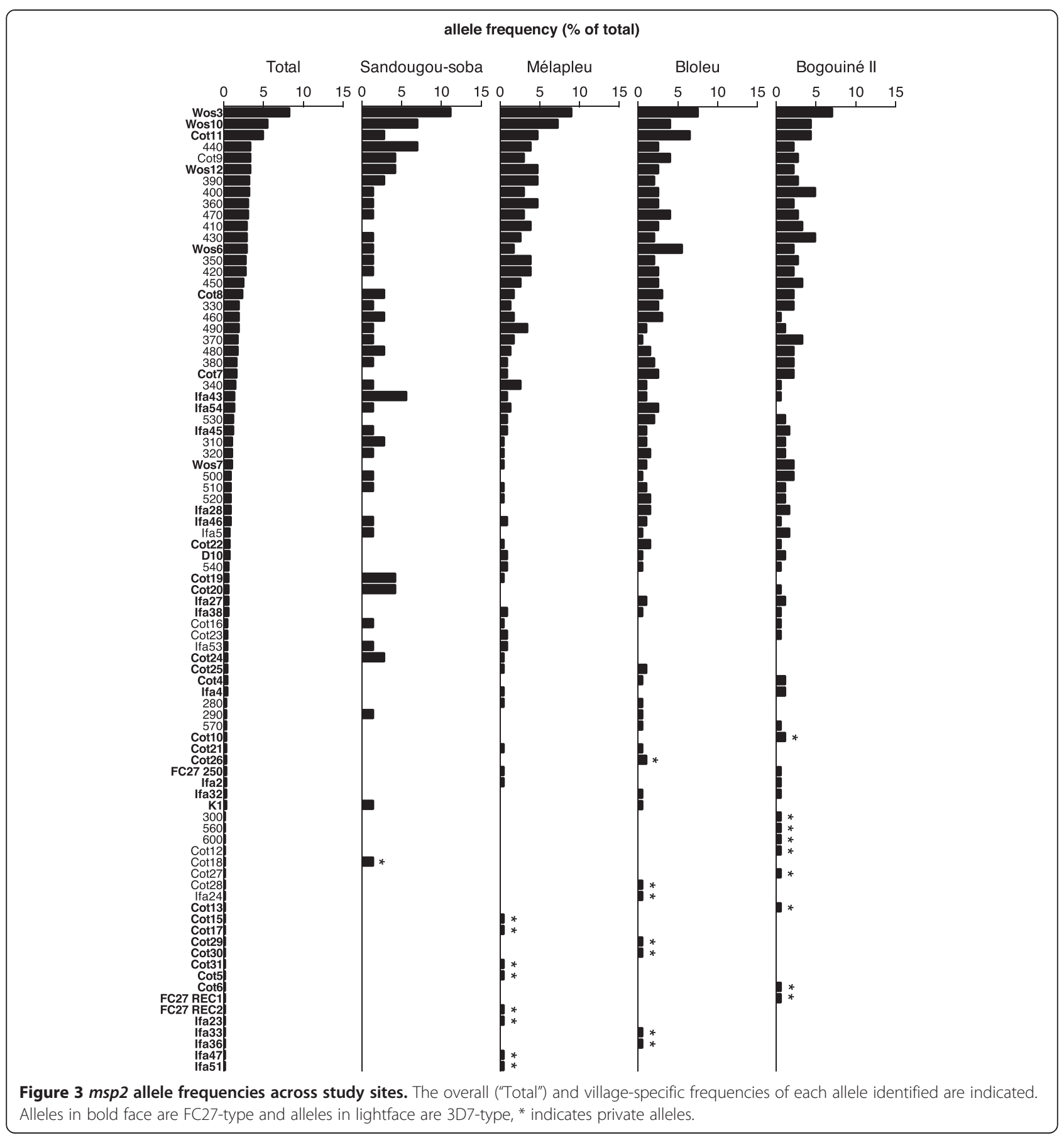

village Bogouiné II, while only 58 alleles were observed from 235 infections in the high altitude village Mélapleu. This suggests that high altitude villages might display a lower diversity than low altitude ones. This observation is further supported by the fact that the number of effective alleles, that is the theoretical number of equally frequent alleles needed to achieve a level of diversity equal to the one observed, is higher in Bogouiné II and Bloleu (low altitude) than in Sandougou-soba and Mélapleu (high altitude), despite the fact that the number of clonal infection sampled is lower in both of these villages as compared to Mélapleu (Table 5).

Nevertheless, the haploid diversity indices were found to be very similar, with values close to 1 in all villages. Similarly, the Shanon's information indices are relatively high, approaching or exceeding 3.5, in all populations. These two indices are indicative of a similarly high degree of diversity and evenness across study locations, suggesting that the local parasite populations investigated are genetically similar and display the same degree of diversity. 
Table 5 Plasmodium falciparum genetic diversity at the study sites

\begin{tabular}{llllll}
\hline Village & $\mathbf{n}$ & $\mathbf{N a}^{\mathbf{a}}$ & $\mathbf{N e}^{\mathbf{b}}$ & $\mathbf{I}^{\mathbf{c}}$ & $\mathbf{h}^{\mathbf{d}}$ \\
\hline Sandougou-Soba & 72 & 39 & 23.78 & 3.427 & 0.958 \\
Mélapleu & 235 & 58 & 27.41 & 3.614 & 0.964 \\
Bloleu & 201 & 58 & 33.20 & 3.753 & 0.970 \\
Bogouiné II & 186 & 60 & 36.34 & 3.816 & 0.972 \\
Total & 694 & 85 & $\mathrm{n} / \mathrm{a}$ & $\mathrm{n} / \mathrm{a}$ & $\mathrm{n} / \mathrm{a}$ \\
Mean & $\mathrm{n} / \mathrm{a}$ & $\mathrm{n} / \mathrm{a}$ & 30.18 & 3.652 & 0.966 \\
SD & $\mathrm{n} / \mathrm{a}$ & $\mathrm{n} / \mathrm{a}$ & 2.82 & 0.086 & 0.003 \\
\hline
\end{tabular}

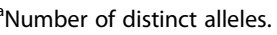

${ }^{b}$ Number of effective alleles.

'Shannon's information index.

${ }^{\mathrm{d}}$ Haploid genetic diversity.

The specific geographic locations of the four study sites was further explored by grouping Sandougou-soba and Mélapleu as villages in the "mountainous" part of the Man region and Bogouiné II and Bloleu in the "plain" for a nested two-setting analysis of molecular variance (AMOVA) (Table 6). The msp2 genetic diversity variations in the different villages were not found to be statistically different $(\Phi P T=0)$, however, a low percentage of diversity variation occurring between the high and low altitude settings was found to be nearing statistical significance $(\Phi \mathrm{RT}=0.001, p=0.07)$, suggesting that parasite populations might not be fully similar between these two settings.

\section{Discussion}

A detailed molecular profiling and genetic diversity analysis of $P$. falciparum in four villages of a hyperendemic area of western Côte d'Ivoire is presented. The study sites were chosen for their relative closeness and contrasted geographical settings, with two villages located in a plain area and two in mountainous area. The P. falciparum prevalence, as determined by PCR, was found to be close to that previously reported by microscopy and above $85 \%$ in all villages except Sandougou-soba, the most elevated village, where it was measured to be $57.4 \%$ and where the parasitaemia GM was also found to be significantly lower. Importantly, the average age of participating children, a potential confounding factor for estimating P. falciparum prevalence and parasitaemia, is comparable from one village to another (Table 1), suggesting that geographical factors might be underlying these differences in the local parasitological pictures. This is in line with several previous studies reporting reduced malaria prevalence at higher altitude, with African highlands, at elevation of 1,500 m above sea level and higher, being generally malaria free but subject to epidemics [34-36]. Lower temperatures at high altitude reduce the development rates of both mosquitoes and Plasmodium parasites, preventing optimal transmission. At intermediate altitude, the fact that hilly environments are less susceptible to stagnant water bodies, which are potential mosquito breeding sites, might also play a role in locally reduced transmission [34]. It is, however, not clear whether these, or other factors, are responsible for the lower malaria prevalence and parasitaemia levels observed in Sandougou-soba.

The genetic diversity of the $P$. falciparum populations was characterized using $m s p 2$ typing, whose higher discriminatory power, as compared to other polymorphic genes, is particularly relevant in hyperendemic areas [17]. The overall theoretical probability to have two clones sharing the same genotype in this study is of $3.4 \%$, which is below the maximal value of $5 \%$ recommended by others [37], and warrants the use of $m s p 2$ as a single marker for genotyping in this context. The genotyping methodology employed in the current study is based on electrophoresis in polyacrylamide gels coupled with computer-assisted fragment sizing using free software. This approach is well adapted to resource-limited settings and was found to be only marginally less precise than more costly methods based on capillary electrophoresis [17,38].

A total of 85 distinct $m s p 2$ alleles were found in the current study and revealed 25 alleles not yet reported elsewhere, expending on a previous study of $m s p 2$ genetic diversity in Côte d'Ivoire [32]. Two newly identified FC27 alleles, Cot13 and Cot14, display deletion in the 137 bp fragment, while an insertion is apparent in Cot31, possibly due the amplification of a 9 bp unit [39]. Some of the potentially new 3D7-type allele display an unusual fragment of approximately 45 bp (Cot18, Cot27 and Cot28), which might be similar to the $51 \mathrm{bp}$ fragment previously observed in Tanzania [40]. Altogether, the limit in precision for fragment size determination does not exclude that

Table 6 Two-setting nested analysis of molecular variance (AMOVA)

\begin{tabular}{llllll}
\hline AMOVA analysis $^{c}$ & df $^{\mathbf{a}}$ & $\mathbf{M S}^{\mathbf{b}}$ & $\%$ of total variation & $\phi$ & $\boldsymbol{\phi}$ \\
\hline Among settings $^{c}$ & 1 & 0.619 & $0.13 \%$ & $\phi$ RT 0.001 & 0.070 \\
Among populations within settings & 2 & 0.418 & $0.00 \%$ & $\phi$ PR -0.001 & 0.785 \\
Among all populations & 80 & 0.486 & $99.87 \%$ & $\phi$ PT 0.000 & 0.275 \\
\hline
\end{tabular}

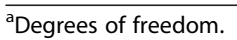

${ }^{\mathrm{b}}$ Mean squared deviations.

"Sandougou-soba and Mélapleu are grouped in a "mountainous" setting, Bogouiné II and Bloleu in a "plain" setting 
some of these potentially new alleles are similar to the ones already described in the literature (e.g. Cot27 appears relatively similar to Ifa6; Cot31 to Ifa34) and sequencing would be required to univocally characterize these $m s p 2$ alleles. Altogether, Wos3 is the most prevalent allele, similarly to what has been observed in south-central Côte d'Ivoire, and two of the newly described alleles in this earlier study were also observed here (Cot5 and Cot6) at low frequencies [32].

Apparent MOI is often used as surrogate for transmission, with higher multiplicity of infections observed in those areas where transmission is particularly high [16]. Consequently, correlations between MOI and parasitaemia or age have been reported [11,17,32,41-43]. In these studies, higher MOI are associated with higher parasitaemia and with younger age, possibly due to lower semiimmunity [44]. The complex interplay between MOI, parasitaemia, age and transmission is not fully understood and other studies failed to report correlations between these parameters, suggesting setting-specific relationships [9,45-47]. In the present investigation, significant differences in MOI averages across the four villages were observed, but with no apparent link with $P$. falciparum prevalence, parasitaemia, village altitude or sampling date (Table 1), the last being a potential confounding factor. A low multiplicity was found in Sandougou-soba, where the lowest prevalence and average parasitaemia were observed, but an equally low multiplicity was also apparent in Bogouiné II, where these parameters were comparatively higher. The factors involved that might explain local differences in MOI are currently not understood. When considering the four villages together, no clear relation was found between age and MOI and only a limited relation with parasitaemia. It appears that in the context of this study, no clear link can be made between MOI and $P$. falciparum prevalence or parasitaemia and, by extension, transmission or with altitude. This is contrasting with a previous study in Kenya, where highland infections display a significantly lower average MOI as compared with lowland areas [36]. The reasons for this difference are unknown and the intrinsic limitations of the current MOI measurements, which are by definition underestimating the real multiplicity of infection, due to clone fluctuation, parasite sequestration in deep vasculature, failure to distinguish different clones or true multiple infections by similar clones, might play a role. Nevertheless, these limitations would be expected to operate equally in all villages and therefore to bias absolute values but not relative comparison.

The $m s p 2$ typing data were also used to evaluate the genetic diversity present in each of the study sites. It can be expected that lower transmission, observed at higher altitude, might result in lower genetic diversity. This hypothesis is supported by the lower number of effective alleles found at higher altitudes. This value can be biased by the sample size, so the most relevant comparison occurs between Bloleu (low altitude) and Mélapleu (high altitude), where an equal number of distinct alleles were observed out of a similar number of infections but with a marked difference in the number of effective alleles, that is 33.2 and 27.4, respectively. A similar trend can be observed, but to a much lower extent, when considering other measures of diversity, such as Shannon's information index and the haploid genetic diversity, which is nearing its maximum value of one in all villages, similar to values previously observed in subSaharan Africa [11]. A low but close to significance degree of genetic variance is apparent between villages of plain and mountainous areas, suggesting the existence of a partially effective partition between the parasite populations of these locations. This is contrasting with a similar study conducted in Uganda, where no difference in strain distribution was observed when comparing villages located in an area of the same scale as the one considered here [46]. The current analysis is intrinsically limited by the fact that only one locus was investigated. It is conceivable that the typing of additional loci, including microsatellites, might have revealed stronger genetic differences. Moreover, the fact that $m s p 2$ is under immune selection might partially mask the true genetic diversity of the populations investigated here. It would also be important to evaluate the temporal evolution of the genetic structures, as time has been identified as a diversity factor almost as important than geographical location in various transmission settings $[15,48]$.

\section{Conclusion}

The current study revealed marked differences in P. falciparum epidemiological and genetic factors within a context of hyperendemicity. Significant differences in prevalence and parasitaemia as well as signs of population partitions could be observed within a distance of approximately $120 \mathrm{~km}$. The local topography in the Man region might partially govern these differences, suggesting the existence of geographical barriers susceptible to locally isolated parasite populations in Côte d'Ivoire. It would be interesting to measure the current genetic diversity in the four study villages in order to evaluate the evolution of the diversity over the past decade. The malERA consortium has highlighted the need for better tools and maps to guide malaria elimination [23], and the identification of natural or human-related barriers defining distinct or partially distinct populations will contribute to address this need. The identification of partially isolated parasite populations might be relevant to guide local malaria elimination intervention when a geographically limited area is targeted. 


\section{Additional files}

\section{Additional file 1: PCR and microscopy derived prevalence.}

(A) P. falciparum detection by PCR and microscopy-based observation of thick smears. The overall higher prevalence detected by PCR $(82.5 \%$ versus $78.8 \%$ ) is due to 27 samples found positive by PCR and negative by microscopy, while 16 samples positive by microscopy were found negative by PCR. (B) Parasitaemia, as determined by microscopy, of the PCR negative $(n=16)$ and PCR positive $(n=214)$ samples. The geometric mean of the PCR negative samples is significantly lower than that of the PCR positive samples (70.3 versus $356.4, p<0.01$, unpaired one-tailed Welch's t-test), suggesting slide reading errors or $P$. falciparum DNA degradation.

Additional file 2: $m s p 2$ genotyping precision. (A) Average, standard deviation and range of the observed sizes of fixed-size msp2 restriction digest products. (B) Linear regression between the observed and expected sizes of the fixed-size msp2 restriction digest products. The average, standard deviation, miminum and maximum value are reported.

Additional file 3: Relation between $\mathrm{MOI}$ and age. (A) Individual $\mathrm{MOI}$ grouped by age clusters (7 to 10 and 11 to 15 years old) in the four study villages. The red lines indicate the means and standard deviations. (B) The average MOls are not significantly different in the two age clusters in each of the four study villages.

\section{Competing interests}

This study received financial support from the Swiss Tropical and Public Health Institute and the Centre Suisse de Recherches Scientifiques en Côte d'lvoire. The funders had no role in the study design, data collection and analysis, decision to publish, or preparation of the manuscript. All authors declare that they have no competing interests.

\section{Authors' contributions}

SEM performed the genotyping and statistical analyses and wrote the first draft of the manuscript. KDS collected the samples, conceived and performed the genotyping analyses and contributed to the writing of the manuscript. GR collected the samples, conceived the genotyping analyses, and contributed to the statistical analyses and to writing of the manuscript. SPN was responsible for the supervision of SEM. EKN, MT, and JU conceived the larger study within which the current genotyping profiling was pursued, were responsible for the supervision of KDS and GR and assisted in the revision of the manuscript. XCD conceived and performed the genotyping analyses and contributed to the statistical analyses and to the writing and revisions of the manuscript. All authors read and approved the final manuscript.

\section{Acknowledgements}

We thank the local health and education authorities, the parents/guardians and the participating children from whom the samples were obtained. We are grateful to M Traoré for the re-examination of a subset of the Giemsastained thick and thin blood films from the original study. We acknowledge the scientific support of I Felger as well as the technical support of T Voss with the PCR-RFLP analyses. We are also thankful to H-P Beck for critical reading of the manuscript.

\section{Author details}

${ }^{1}$ Unité de Formation et de Recherche Biosciences, Université Félix Houphouët-Boigny, 01 BP V34 Abidjan 01, Côte d'Ivoire. ²Département Environnement et Santé, Centre Suisse de Recherches Scientifiques en Côte d'Ivoire, 01 BP 1303 Abidjan 01, Côte d'Ivoire. ${ }^{3}$ Department of Epidemiology and Public Health, Swiss Tropical and Public Health Institute, P.O. Box, CH-4002, Basel, Switzerland. ${ }^{4}$ University of Basel, P.O. Box, CH-4003, Basel, Switzerland.

Received: 5 August 2013 Accepted: 12 November 2013 Published: 15 November 2013

\section{References}

1. Day KP, Koella JC, Nee S, Gupta S, Read AF: Population genetics and dynamics of plasmodium falciparum: an ecological view. Parasitology 1992, 104(Suppl):S35-S52.
2. Manske M, Miotto O, Campino S, Auburn S, Almagro-Garcia J, Maslen G, O'Brien J, Djimdé A, Doumbo O, Zongo I, Ouédraogo JB, Michon P, Mueller I, Siba P, Nzila A, Borrmann S, Kiara SM, Marsh K, Jiang H, Su X-Z, Amaratunga C, Fairhurst R, Socheat D, Nosten F, Imwong M, White NJ, Sanders M, Anastasi E, Alcock D, Drury $E$, et al: Analysis of plasmodium falciparum diversity in natural infections by deep sequencing. Nature 2012, 487:375-379.

3. Anderson TJ, Haubold B, Williams JT, Estrada-Franco JG, Richardson L, Mollinedo R, Bockarie M, Mokili J, Mharakurwa S, French N, Whitworth J, Velez ID, Brockman AH, Nosten F, Ferreira MU, Day KP: Microsatellite markers reveal a spectrum of population structures in the malaria parasite plasmodium falciparum. Mol Biol Evol 2000, 17:1467-1482.

4. Mobegi VA, Loua KM, Ahouidi AD, Satoguina J, Nwakanma DC, Amambua-Ngwa A, Conway DJ: Population genetic structure of plasmodium falciparum across a region of diverse endemicity in West Africa. Malar J 2012, 11:223.

5. Felger I, Genton B, Smith T, Tanner M, Beck H-P: Molecular monitoring in malaria vaccine trials. Trends Parasitol 2003, 19:60-63.

6. Viriyakosol S, Siripoon N, Petcharapirat C, Petcharapirat P, Jarra W, Thaithong $\mathrm{S}$, Brown KN, Snounou G: Genotyping of Plasmodium falciparum isolates by the polymerase chain reaction and potential uses in epidemiological studies. Bull World Health Org 1995, 73:85-95.

7. Creasey A, Fenton B, Walker A, Thaithong S, Oliveira S, Mutambu S, Walliker $D$ : Genetic diversity of plasmodium falciparum shows geographical variation. Am J Trop Med Hyg 1990, 42:403-413.

8. Hoffmann EH, da Silveira LA, Tonhosolo R, Pereira FJ, Ribeiro WL, Tonon AP, Kawamoto F, Ferreira MU: Geographical patterns of allelic diversity in the plasmodium falciparum malaria-vaccine candidate, merozoite surface protein-2. Ann Trop Med Parasitol 2001, 95:117-132.

9. Sakihama N, Nakamura M, Palanca AA, Argubano RA, Realon EP, Larracas AL, Espina RL, Tanabe K: Allelic diversity in the merozoite surface protein 1 gene of plasmodium falciparum on Palawan Island, the Philippines. Parasitol Int 2007, 56:185-194.

10. Ferreira MU, Hartl DL: Plasmodium falciparum: worldwide sequence diversity and evolution of the malaria vaccine candidate merozoite surface protein-2 (MSP-2). Exp Parasitol 2007, 115:32-40.

11. Mwingira F, Nkwengulila G, Schoepflin S, Sumari D, Beck H-P, Snounou G, Felger I, Olliaro P, Mugittu K: Plasmodium falciparum msp1, msp2 and glurp allele frequency and diversity in sub-Saharan Africa. Malar $\lrcorner$ 2011, 10:79.

12. Kyes S, Harding R, Black G, Craig A, Peshu N, Newbold C, Marsh K: Limited spatial clustering of individual plasmodium falciparum alleles in field isolates from coastal Kenya. Am J Trop Med Hyg 1997, 57:205-215.

13. Konaté L, Zwetyenga J, Rogier C, Bischoff E, Fontenille D, Tall A, Spiegel A, Trape JF, Mercereau-Puijalon O: Variation of plasmodium falciparum msp1 block 2 and msp2 allele prevalence and of infection complexity in two neighbouring Senegalese villages with different transmission conditions. Trans R Soc Trop Med Hyg 1999, 93(Suppl 1):21-28.

14. Soulama I, Nébié I, Ouédraogo A, Gansané A, Diarra A, Tiono AB, Bougouma EC, Konaté AT, Kabré GB, Taylor WR, Sirima SB: Plasmodium falciparum genotypes diversity in symptomatic malaria of children living in an urban and a rural setting in Burkina Faso. Malar J 2009, 8:135.

15. Baruah S, Lourembam SD, Sawian CE, Baruah I, Goswami D: Temporal and spatial variation in MSP1 clonal composition of plasmodium falciparum in districts of Assam, Northeast India. Infect Genet Evol 2009, 9:853-859.

16. Kiwanuka GN: Genetic diversity in plasmodium falciparum merozoite surface protein 1 and 2 coding genes and its implications in malaria epidemiology: a review of published studies from 1997-2007. J Vector Borne Dis 2009, 46:1-12.

17. Schoepflin S, Valsangiacomo F, Lin E, Kiniboro B, Mueller I, Felger I: Comparison of plasmodium falciparum allelic frequency distribution in different endemic settings by high-resolution genotyping. Malar J 2009, 8:250.

18. Raso G, Matthys B, N'goran EK, Tanner M, Vounatsou P, Utzinger J: Spatial risk prediction and mapping of Schistosoma mansoni infections among schoolchildren living in western Côte d'Ivoire. Parasitology 2005, 131:97-108.

19. Raso G, Vounatsou P, Gosoniu L, Tanner M, N'goran EK, Utzinger J: Risk factors and spatial patterns of hookworm infection among schoolchildren in a rural area of western Côte d'Ivoire. Int J Parasitol 2006, 36:201-210

20. Silue KD, Raso G, Yapi A, Vounatsou P, Tanner M, N'goran EK, Utzinger J: Spatially-explicit risk profiling of plasmodium falciparum infections at a small scale: a geostatistical modelling approach. Malar J 2008, 7:111. 
21. Raso G, Silue KD, Vounatsou P, Singer BH, Yapi A, Tanner M, Utzinger J, N'goran EK: Spatial risk profiling of plasmodium falciparum parasitaemia in a high endemicity area in Côte d'Ivoire. Malar J 2009, 8:252

22. Raso G, Luginbuhl A, Adjoua CA, Tian-Bi NT, Silue KD, Matthys B, Vounatsou P, Wang Y, Dumas M-E, Holmes E, Singer BH, Tanner M, N'goran EK, Utzinger J: Multiple parasite infections and their relationship to self-reported morbidity in a community of rural Côte d'Ivoire. Int J Epidemiol 2004, 33:1092-1102.

23. Alonso PL, Brown G, Arevalo-Herrera M, Binka F, Chitnis C, Collins F, Doumbo OK, Greenwood B, Hall BF, Levine MM, Mendis K, Newman RD, Plowe $\mathrm{CV}$, Rodríguez $\mathrm{MH}$, Sinden $\mathrm{R}$, Slutsker $\mathrm{L}$, Tanner M: A research agenda to underpin malaria eradication. PLoS Med 2011, 8:e1000406.

24. GPS visualizer. http://www.gpsvisualizer.com.

25. Beck-Wörner C, Raso G, Vounatsou P, N'goran EK, Rigo G, Parlow E, Utzinger $\mathrm{J}$ : Bayesian spatial risk prediction of Schistosoma mansoni infection in western Côte d'Ivoire using a remotely-sensed digital elevation model. Am J Trop Med Hyg 2007, 76:956-963.

26. Foley M, Ranford-Cartwright LC, Babiker HA: Rapid and simple method for isolating malaria DNA from fingerprick samples of blood. Mol Biochem Parasitol 1992, 53:241-244.

27. Felger I, Tavul L, Beck HP: Plasmodium falciparum: a rapid technique for genotyping the merozoite surface protein 2. Exp Parasitol 1993, 77:372-375.

28. Felger I, Beck H-P: Genotyping of plasmodium falciparum. PCR-RFLP analysis. Methods Mol Med 2002, 72:117-129.

29. Schneider CA, Rasband WS, Eliceiri KW: NIH Image to ImageJ: 25 years of image analysis. Nat Methods 2012, 9:671-675.

30. Peakall R, Smouse PE: GENALEX 6: genetic analysis in excel. Population genetic software for teaching and research. Mol Ecol Notes 2006, 6:288-295.

31. Peakall R, Smouse PE: GenAIEx 6.5: genetic analysis in excel. Population genetic software for teaching and research-an update. Bioinformatics 2012, 28:2537-2539.

32. Silue KD, Felger I, Utzinger J, Beck HP, Smith TA, Tanner M, N'goran EK: [Prevalence, genetic diversity and multiplicity of plasmodium falciparum infection in school children in central cote d'Ivoire]. Med Trop (Mars) 2006, 66:149-156.

33. Leberg PL: Estimating allelic richness: effects of sample size and bottlenecks. Mol Ecol 2002, 11:2445-2449.

34. Lindsay SW, Martens WJ: Malaria in the African highlands: past, present and future. Bull World Health Org 1998, 76:33-45.

35. Drakeley CJ, Carneiro I, Reyburn H, Malima R, Lusingu JPA, Cox J, Theander TG, Nkya WMMM, Lemnge MM, Riley EM: Altitude-dependent and -independent variations in plasmodium falciparum prevalence in northeastern Tanzania. J Infect Dis 2005, 191:1589-1598.

36. Zhong D, Afrane Y, Githeko A, Yang Z, Cui L, Menge DM, Temu EA, Yan G: Plasmodium falciparum genetic diversity in western Kenya highlands. Am J Trop Med Hyg 2007, 77:1043-1050.

37. Gatton ML, Cheng Q: Can estimates of antimalarial efficacy from field studies be improved? Trends Parasitol 2008, 24:68-73.

38. Falk N, Maire N, Sama W, Owusu-Agyei S, Smith T, Beck H-P, Felger I: Comparison of PCR-RFLP and Genescan-based genotyping for analyzing infection dynamics of plasmodium falciparum. Am J Trop Med Hyg 2006, 74:944-950

39. Irion A, Beck HP, Felger I: New repeat unit and hot spot of recombination in FC27-type alleles of the gene coding for plasmodium falciparum merozoite surface protein 2. Mol Biochem Parasitol 1997, 90:367-370.

40. Felger I, Irion A, Steiger S, Beck HP: Genotypes of merozoite surface protein 2 of plasmodium falciparum in Tanzania. Trans $R$ Soc Trop Med Hyg 1999, 93(Suppl 1):3-9.

41. Felger I, Smith T, Edoh D, Kitua A, Alonso P, Tanner M, Beck HP: Multiple plasmodium falciparum infections in Tanzanian infants. Trans R Soc Trop Med Hyg 1999, 93(Suppl 1):29-34.

42. Jordan S, Jelinek T, Aida AO, Peyerl-Hoffmann G, Heuschkel C, Valy el AO, Christophel EM: Population structure of plasmodium falciparum isolates during an epidemic in southern Mauritania. Trop Med Int Health 2001, 6:761-766.

43. Mayor A, Saute F, Aponte JJ, Almeda J, Gómez-Olivé FX, Dgedge M, Alonso PL: Plasmodium falciparum multiple infections in Mozambique, its relation to other malariological indices and to prospective risk of malaria morbidity. Trop Med Int Health 2003, 8:3-11.

44. Hviid L, Staalsoe T: Malaria immunity in infants: a special case of a general phenomenon? Trends Parasitol 2004, 20:66-72
45. Engelbrecht F, Tögel E, Beck HP, Enwezor F, Oettli A, Felger I: Analysis of plasmodium falciparum infections in a village community in Northern Nigeria: determination of msp2 genotypes and parasite-specific lgG responses. Acta Trop 2000, 74:63-71.

46. Peyerl-Hoffmann G, Jelinek T, Kilian A, Kabagambe G, Metzger WG, Sonnenburg von F: Genetic diversity of plasmodium falciparum and its relationship to parasite density in an area with different malaria endemicities in West Uganda. Trop Med Int Health 2001, 6:607-613.

47. Aubouy A, Migot-Nabias F, Deloron P: Polymorphism in two merozoite surface proteins of plasmodium falciparum isolates from Gabon. Malar $\rfloor$ 2003, 2:12.

48. Branch OH, Sutton PL, Barnes C, Castro JC, Hussin J, Awadalla P, Hijar G: Plasmodium falciparum genetic diversity maintained and amplified over 5 years of a low transmission endemic in the Peruvian Amazon. Mol Biol Evol 2011, 28:1973-1986.

doi:10.1186/1475-2875-12-419

Cite this article as: Mara et al:: Genetic diversity of Plasmodium falciparum among school-aged children from the Man region, western Côte d'Ivoire. Malaria Journal 2013 12:419.

\section{Submit your next manuscript to BioMed Central and take full advantage of:}

- Convenient online submission

- Thorough peer review

- No space constraints or color figure charges

- Immediate publication on acceptance

- Inclusion in PubMed, CAS, Scopus and Google Scholar

- Research which is freely available for redistribution 